\title{
X-rays from young stars - a summary of highlights from the XMM-Newton Extended Survey of the Taurus Molecular Cloud (XEST)
}

\author{
M. Güdel ${ }^{1,2}$ \\ ${ }^{1}$ Paul Scherrer Institut, Würenlingen \& Villigen, CH-5232 Villigen PSI, Switzerland; guedel@ astro.phys.ethz.ch \\ ${ }^{2}$ Leiden Observatory, Leiden University, PO Box 9513, 2300 RA Leiden, The Netherlands
}

Key words: stars: activity - stars: formation - stars: pre-main sequence - T Tauri stars - X-rays: stars

Received 26 October 2007; accepted 30 October 2007

\begin{abstract}
The XMM-Newton Extended Survey of the Taurus Molecular Cloud (XEST) is a survey of the nearest large star-forming region, the Taurus Molecular Cloud (TMC), making use of all instruments on board the XMM-Newton X-ray observatory. The survey, presently still growing, has provided unprecedented spectroscopic results from nearly every observed $\mathrm{T}$ Tauri star, and from $\approx 50 \%$ of the studied brown dwarfs and protostars. The survey includes the first coherent statistical sample of high-resolution spectra of $\mathrm{T}$ Tauri stars, and is accompanied by an U-band/ultraviolet imaging photometric survey of the TMC. XEST led to the discovery of new, systematic X-ray features not possible before with smaller samples, in particular the $X$-ray soft excess in classical T Tauri stars and the Two-Absorber X-ray (TAX) spectra of jet-driving T Tauri stars. This paper summarizes highlights from XEST and reviews the key role of this large project.
\end{abstract}

\section{Introduction}

Studies of star-forming regions have drawn a picture in which cool, molecular gas, contracting to stars with surrounding accretion disks, co-exists with high-energy radiation. The latter is emitted by stellar plasma that is continuously heated to temperatures of several million K. This radiation is most prominently seen in the soft $\mathrm{X}$-ray range and is conventionally attributed to the presence of magnetic, coronal plasma.

In contrast to the solar case, high-energy radiation from forming stars has a profound influence on the stellar environment. Extreme ultraviolet and X-ray photons ionize and heat dense gas in accretion disks and the larger-scale molecular envelopes (e.g., Glassgold, Najita, \& Igea 2004). As a consequence, complex chemical networks may be driven. Further, ionized disk surface layers make the gas accessible to magnetic fields, which then induce the magnetorotational instability relevant for the accretion process (Balbus \& Hawley 1991). Ionized and magnetized environments are also important for the disk-star accretion process through magnetic funnels, and for the acceleration of bi-polar jets.

A deeper understanding of these physical mechanisms requires systematic studies of young stellar samples. The project described here has aimed at the nearest large starformation complex, the Taurus Molecular Cloud (TMC), using all science instruments on board XMM-Newton (Jansen et al. 2001).

\section{XEST and the Taurus Molecular Cloud}

The TMC is the closest and best-studied large star formation region; it has served as a test-bed for low-mass starformation theory for decades. The relevant region is quite large, however (some 10-15 degrees in diameter, corresponding to about $25-35 \mathrm{pc}$ at a distance of $140 \mathrm{pc}$ ), making comprehensive studies of the entire population difficult.

The XMM-Newton Extended Survey of the Taurus Molecular Cloud (XEST) concentrates on the denser cloud areas that contain the bulk of the TMC stellar population. The emphasis is on a wide-field survey rather than on long exposures, although the total exposure time has now reached $\approx 1.3$ Ms. XEST goes systematically deeper than previous Taurus surveys by about an order of magnitude. A few exposures obtained by the Chandra X-Ray Observatory have been added to the survey for completeness.

The concept, strategy, and databases of XEST are described in Güdel et al. (2007a); the initial science results have been published in 14 accompanying papers in a special feature of Astronomy \& Astrophysics (Vol. 468, 2007 June $3)$. XEST is a continuing survey, however. Following the initial 27 EPIC fields of view, each with a diameter of 30 ar$\mathrm{cmin}$, four additional exposures have been obtained, and at least three further fields will become available in the archive.

\section{The XEST stellar population}

The detection statistics of our EPIC (Strüder et al. 2001; Turner et al. 2001) X-ray survey is summarized in Table 1 separately for the XMM-Newton data, and for the combined XMM-Newton and Chandra fields (in parentheses). The Xray sample of detected classical TTS (CTTS) and weak-lined TTS (WTTS) is nearly complete for the surveyed areas as far as the population is known. Most of the few remaining, undetected objects are considerably extincted and by implication X-ray absorbed. 
Table 1: XEST X-ray detection statistics of TMC stars and BDs. Numbers in parentheses include Chandra observations.

\begin{tabular}{lrrrrr}
\hline Object type & $\begin{array}{c}\text { Members } \\
\text { surveyed }\end{array}$ & Detections & $\begin{array}{l}\text { Detection } \\
\text { fraction (\%) }\end{array}$ \\
\hline Protostars & 20 & $(21)$ & 8 & $(10)$ & $40 \%(48 \%)$ \\
CTTS & 65 & $(70)$ & 55 & $(60)$ & $85 \%(86 \%)$ \\
WTTS & 50 & $(52)$ & 49 & $(50)$ & $98 \%(96 \%)$ \\
BD & 16 & $(17)$ & 8 & $(9)$ & $50 \%(53 \%)$ \\
Herbig stars & 2 & & 2 & & $100 \%$ \\
others/unident. & 6 & $(7)$ & 4 & $(5)$ & $67 \%(71 \%)$ \\
\hline Total & $159(169)$ & 126 & $(136)$ & $79 \%(80 \%)$ \\
\hline
\end{tabular}

The Optical Monitor (Mason et al. 2001) has detected a total of 2148 point sources mostly in the $U$ band, but partly also in ultraviolet (UV) bands (Audard et al. 2007); of these, 1893 objects have 2MASS counterparts, i.e., are most likely to be stellar. Among them, 51 objects are known stellar/substellar members of TMC with X-ray counterparts.

The Reflection Grating Spectrometers (den Herder et al. 2001) secured sufficiently good spectra of four CTTS, four WTTS, and one Herbig star, making this by far the largest coherent sample of high-resolution X-ray spectra of TTS available at the time (Telleschi et al. 2007b).

\section{Accretion: UV and X-ray signatures}

Accretion is thought to proceed from the inner border of the CTTS accretion disk to the star via magnetospheric accretion funnels, producing shocks near the photospheric layers; the shock-heated gas is detected in large UV excess fluxes (e.g., Johns-Krull et al. 2000). Although the distributions of bolometric (stellar) luminosities of the CTTS and WTTS samples agree with each other, the XEST OM survey indeed revealed, after extinction corrections, a blue-/UV-excess in CTTS by up to 3 magnitudes (Audard et al. 2007).

Variable accretion rates reflect in U-band and UV variability. A particularly striking example was seen in an accreting brown dwarf (BD) in which the $\mathrm{U}$ band magnitude slowly increased during approximately 6 hours while the star remained entirely undetected in X-rays; Grosso et al. (2007b) concluded that the accretion rate increased by a factor of $\approx 3$ during that event.

As this example shows, there is in general no correlation between X-ray and UV excess events, as is also evident in the large XEST collection of simultaneous UV and X-ray light curves; any correlated behavior shows signatures of coronal flares, i.e., the UV event precedes the X-ray flare (Audard et al. 2007; Fig. 1). As a consequence of accretion, however, CTTS are much more UV variable than WTTS.

Are there $X$-ray signatures of accretion? XEST confirmed a general X-ray deficiency by a factor of $\approx 2$ for CTTS compared to WTTS (previously reported for other TTS, see Telleschi et al. 2007a for a summary; Fig. 2), both in the $L_{\mathrm{X}}$ and $L_{\mathrm{X}} / L_{\mathrm{bol}}$ distributions while the distributions of $L_{\mathrm{bol}}$

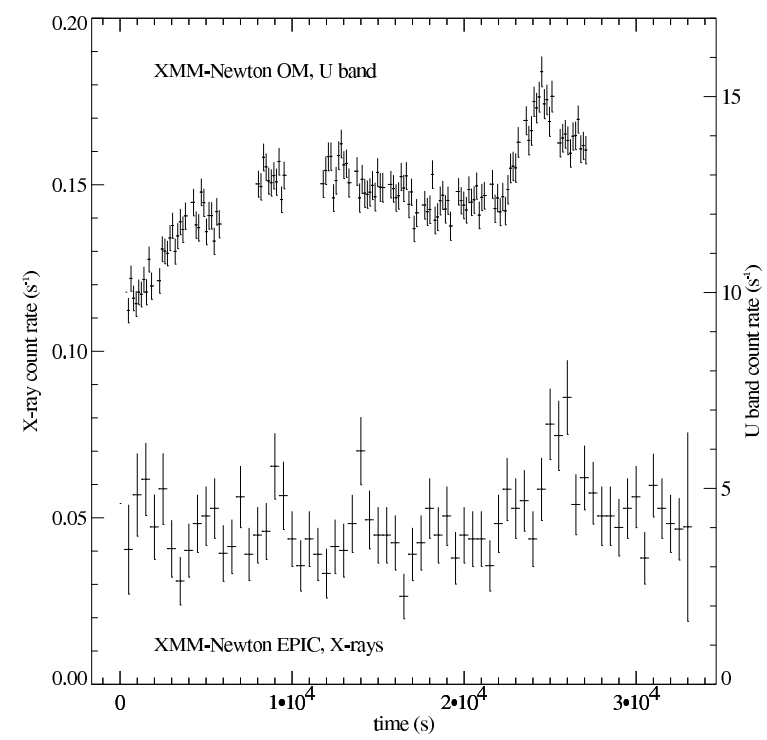

Figure 1: U-band and X-ray light curves of the CTTS GK Tau. The correlated event toward the end of the observation shows signatures of a flare, while the uncorrelated slow variation in the U-band curve is most likely due to variable accretion (after Audard et al. 2007).

for the two samples are indistinguishable. The cause of Xray suppression is not clear but appears to be related to a suppression of the coronal heating efficiency by inflowing cool, accreting material (Preibisch et al. 2005; Telleschi et al. 2007a). The distinction is less clear among BDs; both accreting and non-accreting BDs show similar X-ray luminosity distributions, confirming the predominantly magnetic origin of the X-rays (Grosso et al. 2007a).

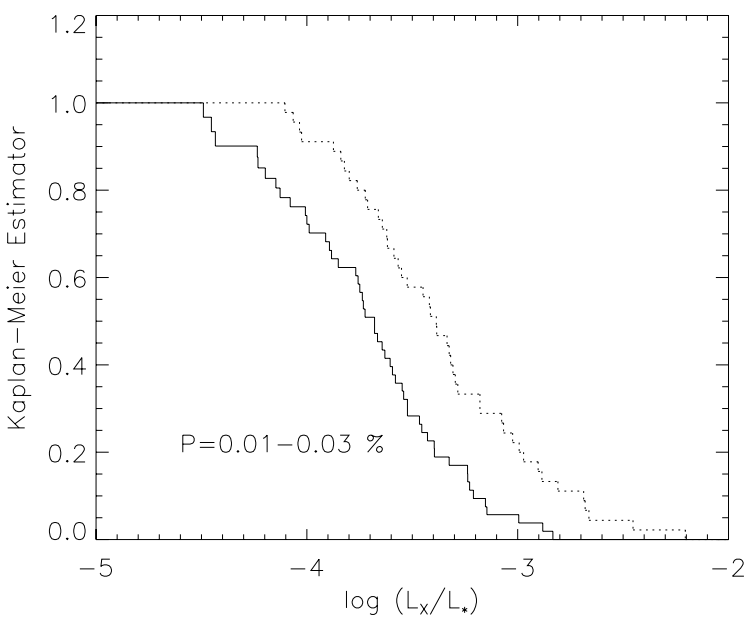

Figure 2: Cumulative distributions (Kaplan-Meier estimator) for fractional X-ray luminosities, $L_{\mathrm{X}} / L_{\mathrm{bol}}$, of classical (solid) and weak-line (dotted) $\mathrm{T}$ Tauri stars in TMC (Telleschi et al. 2007a). 


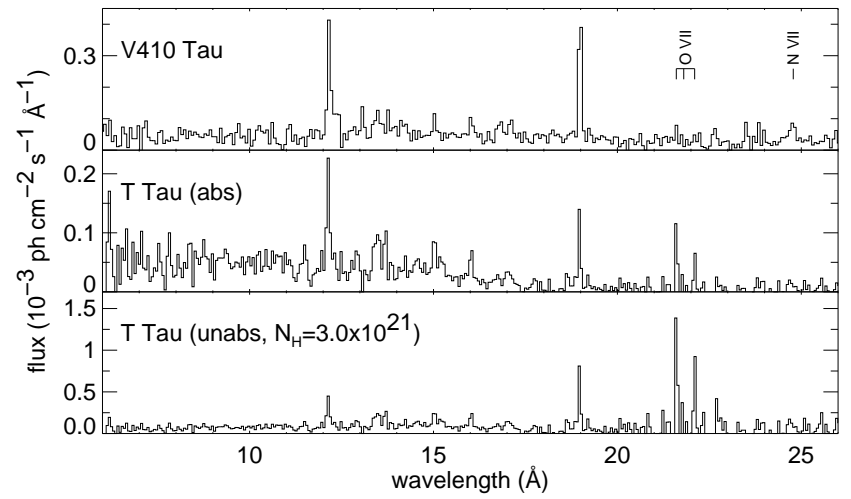

Figure 3: Fluxed X-ray photon spectra of the WTTS V410 Tau, the CTTS T Tau, and the T Tau spectrum modeled after removal of absorption (from top to bottom; Güdel \& Telleschi 2007).

\section{Discovery of the CTTS "X-ray soft excess"}

Nearly free-falling gas will shock-heat to maximum temperatures $T_{s}=8.6 \times 10^{5} \mathrm{~K}\left[M /\left(0.5 M_{\odot}\right)\right]\left[R /\left(2 R_{\odot}\right)\right]^{-1}$ (Calvet $\&$ Gullbring 1998). It is therefore conceivable that the softest $\mathrm{X}$-ray range reveals the high- $T$ tail of the shock emission measure. High electron densities up to $n_{\mathrm{e}} \approx 10^{13} \mathrm{~cm}^{-3}$, measured in density-sensitive X-ray lines of CTTS, have indeed been attributed to shocked gas (Kastner et al. 2002) although the XEST sample is ambiguous in this respect, showing two accretors with low densities (AB Aur: Telleschi et al. 2007c; T Tau: Güdel et al. 2007c).

On the other hand, the unprecedented, large XEST sample of high-resolution RGS spectra revealed a new systematic not accessible before, namely an unusually high flux ratio between the O VII and the O VIII Ly $\alpha$ lines in CTTS. This excess flux, coined the $X$-ray soft excess, was first described by Güdel (2006) and comprehensively discussed by Telleschi et al. (2007b) and Güdel et al. (2007c).

The excessive O VII flux is best illustrated for T Tau when compared to WTTS (or main-sequence [MS] stars), and after removal of the photoelectric absorption in T Tau (Fig. 3). The O VII lines are the strongest in the soft X-ray spectrum of $\mathrm{T}$ Tau, while they normally remain undetected in WTTS. These lines are enhanced by typically a factor of 34 in CTTS compared to WTTS and equivalently active MS stars (Fig. 4). This is different from the UV excesses that are much stronger, and suggests a relation with coronal activity although accretion is conditional for the $X$-ray soft excess. The X-ray soft excess thus is a new accretion diagnostic for TTS discovered in the XEST project.

\section{The "Two-Absorber X-Ray Sources"}

A new type of X-ray spectrum of CTTS was reported by Güdel et al. (2005) and systematically uncovered in several XEST CTTS (Güdel et al. 2007b). The spectra of the TwoAbsorber X-ray (TAX) sources show two unrelated components subject to largely different photoelectric absorption.

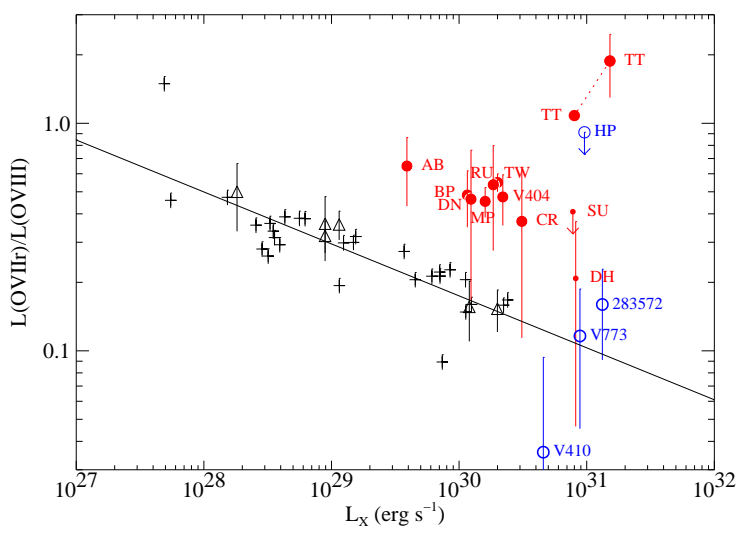

Figure 4: The ratio between O VII $r$ and O VIII Ly $\alpha$ luminosities vs. $L_{\mathrm{X}}$. Crosses mark MS stars, triangles solar analogs, filled (red) circles CTTS, and open (blue) circles WTTS. The solid line is a power-law fit to the MS stars (from Güdel \& Telleschi 2007).

A soft component from a cool (few MK) plasma, subject to very small absorption, is accompanied by a much harder component from a hot (tens of MK) plasma, subject to about ten times higher absorption (Fig. 5). This latter absorption is, for standard interstellar gas-to-dust ratios, excessive if compared to the stellar visual extinction.

The TMC TAX sources (DG Tau, GV Tau, DP Tau, CW Tau - Güdel et al. 2007b - and HN Tau - Fig. (5) have in common i) a high accretion rate, accompanied by a high mass outflow rate, and ii) the presence of "micro-jets". DG Tau's bi-polar jet has meanwhile been identified in X-rays out to a distance of $5^{\prime \prime}$ from the star (Güdel et al. 2008).

The hard component flares in three TAX sources, suggesting a coronal/magnetospheric origin. The excessive absorption is attributed to massive accretion streams; they are, given their close distance to the star, dust depleted, therefore not inducing excessive visual extinction. The absorption of the hard component thus provides unique signatures of accretion streams.

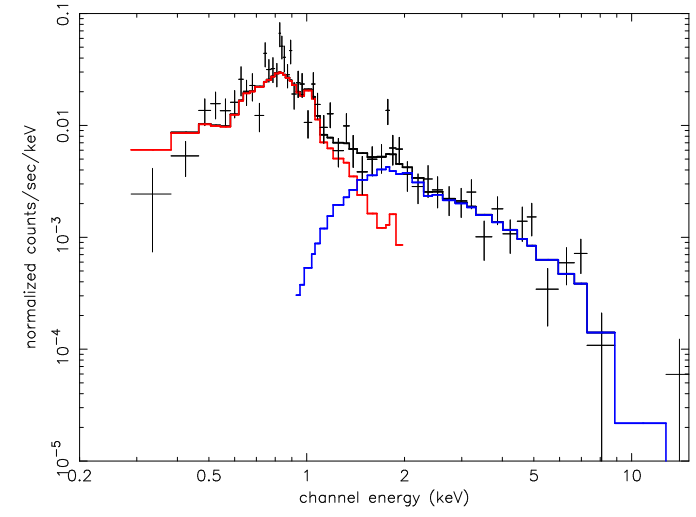

Figure 5: TAX spectrum of HN Tau (Güdel et al. 2008, in prep). 
The non-variable, weakly absorbed soft component cannot originate from the same regions; its close spectral similarity with DG Tau's jet X-ray emission suggests an origin at the base of the jets. These sources may thus directly irradiate circumstellar disks from above.

\section{A new abundance diagnostic?}

Depending on grain evolution in the circumstellar disk, certain elements may condense onto growing solids and will not be accreted onto the star. If accretion feeds the X-ray source with new material, an anomalous composition may result (e.g., Drake, Testa, \& Hartmann 2005). We found no difference between CTTS and WTTS for abundance ratios between $\mathrm{Fe}, \mathrm{Ne}$, and $\mathrm{O}$ determined from high-resolution spectroscopy. However, a dependence on spectral type or $T_{\text {eff }}$ shows up in that the $\mathrm{Fe} / \mathrm{Ne}$ ratio is lower in later-type (K and M) stars than in G-type stars (Telleschi et al. 2007b; Güdel et al. 2007c). This systematic in fact extends to active, "X-ray saturated" MS and post-MS stars as well and is therefore not related to accretion. It has been confirmed for a larger sample of XEST EPIC spectra (Scelsi et al. 2007).

\section{XEST: Successes and expansion}

XEST has been among the first XMM-Newton "Large Programs". The relevance and importance of extended XMMNewton programs is illustrated by systematic XEST findings that were not feasible with the incoherent, smaller samples available before. XEST has specifically succeeded in

- the discovery of the accretion-related X-ray soft excess of CTTS by securing the largest uniform sample of Xray grating spectra of TTS simply not available before;

- the systematic detection of several two-absorber $X$-ray sources now attributed to jets;

- the demonstration of new abundance systematics from $\mathrm{X}$-ray spectroscopy, the $\mathrm{Fe} / \mathrm{Ne}$ abundance ratio depending on spectral type but not on accretion properties;

- a systematic study of the (mostly accretion-related) Uband/UV fluxes in the context of the (mostly coronal) $\mathrm{X}$-rays for a large sample of TTS, revealing the absence of correlated time behavior except during flares;

- the detection of an appreciable sample of X-ray emitting BDs, testifying to the low detection limit of the survey.

Many findings have remained unmentioned in this report, e.g., from studies of variability and flares, rotation, and the search for new TMC members. Clearly, the concept of a large program has been essential for XEST. A collection of restricted, non-uniform small programs conducted by various teams with different goals and instrument settings will not replace a uniformly conducted survey.

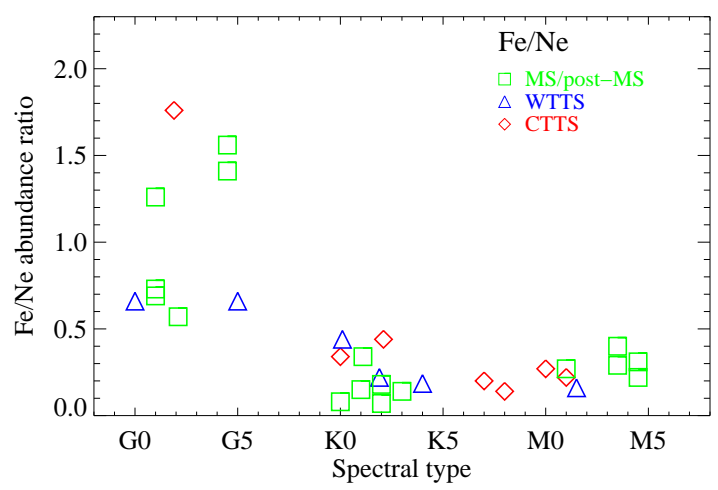

Figure 6: Systematics in the $\mathrm{Fe} / \mathrm{Ne}$ abundance ratio as a function of spectral type; stellar types are as indicated by the symbols and colors. The ratios are relative to the solar photospheric ratio (after Güdel et al. 2007c; Telleschi et al. 2007b).

XEST is growing by several new exposures being added at the time of writing. Four additional exposures have been obtained, and at least three are becoming available in the archive. As far as these and future exposures are compatible with previous exposures of XEST, they will be uniformly reduced and analyzed, and then combined with the published survey. New results will be reported in upcoming papers.

Acknowledgments: We thank the International Space Science Institute (ISSI, Bern, Switzerland) for financial support of XEST. This research is based on observations obtained with XMM-Newton, an ESA science mission with instruments and contributions directly funded by ESA member states and the USA (NASA).

\section{References}

Audard, M., Briggs, K.R., Grosso, N., Güdel, M., Scelsi, L., Bouvier, J., Telleschi, A. 2007: A\&A 468, 379

Balbus, S. A., Hawley J. F.: 1991, ApJ 376, 214

Calvet, N., Gullbring, E.: 1998, ApJ 509, 802

den Herder, J. W., Brinkman, A. C., Kahn, S. M., et al.: 2001, A\&A 365, L7

Drake, J. J., Testa, P., Hartmann, L.: 2005, ApJ 627, L149

Glassgold, A. E., Najita, J., Igea, J.: 2004, ApJ 615, 972

Grosso, N., Audard A., Bouvier, J., Briggs, K. R., Güdel, M.: 2007b, A\&A 468, 557

Grosso, N., Briggs, K. R., Güdel, M., et al.: 2007a, A\&A 468, 391

Güdel, M. 2006, in High-Resolution X-Ray Spectroscopy; ed. G. Branduardi-Raymont, http:// www.mssl.ucl.ac.uk/ gbr/workshop2/; astro-ph/0609281

Güdel, M., Telleschi, A.: 2007, A\&A 474, L25

Güdel, M., Briggs, K. R., Arzner, K., et al. 2007a: A\&A 468, 353

Güdel, M., Skinner, S. L., Audard, M., Briggs, K. R., Cabrit, S.: 2008: A\&A, submitted

Güdel, M., Skinner, S. L., Briggs, K. R., et al.: 2005, ApJ 626, L53

Güdel, M., Skinner, S. L., Mel'nikov, S. Y., Audard, M., Telleschi, A., Briggs, K. R.: 2007c, A\&A 468, 529

Güdel, M., Telleschi, A., Audard, M., Skinner, S. L., Briggs, K. R., Palla, F.: 2007b, A\&A 468, 515 
Jansen, F., Lumb, D., Altieri, B., et al.: 2001, A\&A 365, L1

Johns-Krull, C.M., Valenti, J.A., Linsky, J.L.: 2000, ApJ 539, 815

Kastner, J. H., Huenemoerder, D. P., Schulz, N. S., Canizares, C. R., Weintraub, D. A.: 2002, ApJ 567, 434

Mason, K. O., Breeveld, A., Much, R., et al.: 2001, A\&A 365, L36

Preibisch, T., Kim, Y.-C., Favata, F., et al.: 2005, ApJS 160, 401

Scelsi, L., Maggio, A., Micela, G., Briggs, K. R., Güdel, M.: 2007, A\&A 473, 589

Strüder, L., Briel, U., Dennerl, K., et al.: 2001, A\&A 365, L18

Telleschi, A., Güdel, M., Briggs, K. R., Audard, M., Palla, F.: 2007a, A\&A 468, 425

Telleschi, A., Güdel, M., Briggs, K. R., Audard, M., Scelsi, L.: 2007b, A\&A 468, 443

Telleschi, A., Güdel, M., Briggs, K. R., Skinner, S. L., Audard, M., Franciosini, E.: 2007c, A\&A 468, 541

Turner, M. J. L., Abbey, A., Arnaud, M., et al.: 2001, A\&A 365, L27 